\title{
HIV self-testing and STI self-collection via mobile apps: experiences from two pilot randomized controlled trials of young men who have sex with men
}

\author{
Katie B. Biello, ${ }^{1,2,3}$, Casey Horvitz ${ }^{4}$, Shelby Mullin ${ }^{5}$, Kenneth H. Mayer ${ }^{2,6,7}$, Hyman Scott ${ }^{8,9}$, \\ Kenneth Coleman ${ }^{8}$, Julian Dormitzer ${ }^{2}$, Jenna Norelli ${ }^{5}$, Lisa Hightow-Weidman ${ }^{4}$, Patrick Sullivan ${ }^{5}$, \\ Matthew J. Mimiaga ${ }^{1,2,3,10}$, Susan Buchbinder ${ }^{8,9}$, Kelly Bojan ${ }^{11}$, Donna Futterman ${ }^{12,13}$, Patricia Emmanuel ${ }^{14}$, \\ Albert Liu, ${ }^{8,9}$ on behalf of the iTech Team
}

${ }^{1}$ Departments of Behavioral \& Social Health Sciences and Epidemiology, School of Public Health, Brown University, Providence, RI, USA; ${ }^{2}$ The Fenway Institute, Fenway Health, Boston, MA, USA; ${ }^{3}$ Center for Health Promotion and Health Equity, Brown University, Providence, RI, USA; ${ }^{4}$ Institute for Global Health \& Infectious Diseases, University of North Carolina, Chapel Hill, NC, USA; ${ }^{5}$ Rollins School of Public Health, Emory University, Atlanta, GA, USA; ${ }^{6}$ Department of Global Health and Population, Harvard T.C. Chan School of Public Health, Boston, MA, USA; ${ }^{7}$ Department of Infectious Diseases, Beth Israel Deaconess Medical Center, Boston, MA, USA; ${ }^{8}$ Bridge HIV, San Francisco Department of Public

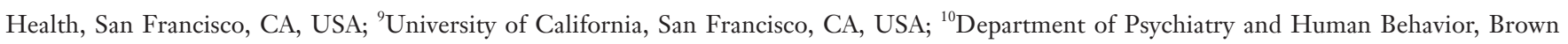
University Alpert Medical School, Providence, RI, USA; ${ }^{11}$ Division of Adolescent Medicine, The Ruth M. Rothstein CORE Center, Chicago, IL, USA; ${ }^{12}$ Adolescent AIDS Program, Children's Hospital at Montefiore, Bronx, NY, USA; ${ }^{13}$ Department of Pediatrics, Albert Einstein College of Medicine, Bronx, NY, USA; ${ }^{14}$ Department of Pediatrics, University of South Florida Morsani College of Medicine, Tampa, FL, USA Contributions: (I) Conception and design: KB Biello, KH Mayer, H Scott, K Coleman, L Hightow-Weidman, P Sullivan, MJ Mimiaga, S Buchbinder, A Liu; (II) Administrative support: J Dormitzer; (III) Provision of study materials or patients: K Bojan, D Futterman, P Emmanuel, J Dormitzer; (IV) Collection and assembly of data: C Horvitz, S Mullin, J Norelli, K Bojan, D Futterman, P Emmanuel, J Dormitzer; (V) Data analysis and interpretation: KB Biello, C Horvitz, S Mullin, A Liu; (VI) Manuscript writing: All authors; (VII) Final approval of manuscript: All authors.

Correspondence to: Katie B. Biello, PhD, MPH. Associate Professor, Departments of Behavioral \& Social Sciences and Epidemiology, Center for Health Promotion and Health Equity, Brown University School of Public Health, Box G-S121-8, Providence, RI 02912, USA.

Email: katie_biello@brown.edu.

Background: Young men who have sex with men (YMSM) are disproportionately impacted by HIV and other sexually transmitted infections (STIs) in the United States (US) and have low rates of HIV/STI testing. Provision of HIV self-testing and STI self-collection can increase testing rates, and access to these kits through mobile applications (apps) could help facilitate YMSM using HIV self-testing and STI selfcollection.

Methods: Data for this study comes from two pilot randomized controlled trials (RCTs) of mobile apps within the Adolescent Trials Network-LYNX and MyChoices—aimed to increase HIV/STI testing among YMSM (age 15-24) who had not recently tested for HIV and were at high risk for HIV acquisition across five US cities. Both apps include the ability to order a HIV self-test with rapid results and a kit for STI self-collection and mailing of samples for syphilis, gonorrhea and chlamydia to a lab for testing. Using assessments of app users $(n=80)$ at pre-randomization and at 3- and 6-months post-randomization and online interview data from a purposive sample of app users $(n=37)$, we report on experiences and lessons learned with HIV self-testing and STI self-collection kits ordered via the apps.

Results: Participants were on average 20.7 years of age (SD =2.4), and 49\% were non-White or multiple race/ethnicity. Sixty-three percent had a prior HIV test. Over half (58\%) had a prior STI test, but only $3 \%$ had tested within the past 3 months. Nearly two-thirds ordered an HIV self-testing kit; of whom, 75\% reported using at least one self-test kit over the study period. STI self-collection kit ordering rates were also high (54\%); however, STI self-collection kit return rates were lower (13\%), but with a high positivity rate (5.3\%). Both HIV self-testing and STI self-collection kits were highly acceptable, and $87 \%$ reported that it was extremely/very helpful to be able to order these kits through the apps. The most common reason for 
not ordering the HIV/STI kits was preferring to test at a clinic. In interviews, participants expressed feeling empowered by being able to test at home; however, they also raised concerns around STI sample collection.

Conclusions: HIV self-testing and STI self-collection kit ordering via mobile apps is feasible, acceptable and may show promise in increasing testing rates among YMSM. The LYNX and MyChoices apps are currently being tested in a full-scale efficacy trial, and if successful, these innovative mobile apps could be scaled up to efficiently increase HIV/STI testing among youth across the US.

Keywords: Self-testing; HIV; sexually transmitted infections (STIs); mobile apps; young men who have sex with men (YMSM)

Received: 20 February 2020; Accepted: 15 July 2020; Published: 20 April 2021.

doi: $10.21037 /$ mhealth-20-70

View this article at: http://dx.doi.org/10.21037/mhealth-20-70

\section{Introduction}

Young men who have sex with men (YMSM) are disproportionately impacted by HIV in the United States (US), with youth aged $13-24$ accounting for $21 \%$ of new HIV diagnoses in 2017 , and $81 \%$ of these occurring among YMSM (1). YMSM of color are particularly impacted, with $51 \%$ and $25 \%$ of new diagnoses occurring in Black and Latinx YMSM, respectively. Furthermore, only $56 \%$ of youth living with HIV are aware of their infection, the lowest proportion among any age group, and youth are the least likely to be linked to care and have a suppressed viral load (1-4). Additionally, YMSM have disproportionately high rates of bacterial sexually transmitted infections (STIs) (5), with rates of chlamydia, gonorrhea, and syphilis increasing $28-55 \%$ among young men from 2013 to 2017 (6).

Although the Centers for Disease Control and Prevention (CDC) recommends at least yearly HIV testing for MSM (7), HIV testing rates among YMSM remain low. In the Youth Risk Behavioral Surveillance System, only 17\% of sexually active male high school students had ever tested, and among young adult males aged $18-24$, only $27 \%$ had ever been tested (8). In a recent national online survey, only $45 \%$ of YMSM reported testing in the past year, and $42 \%$ had never tested in their lifetime (9). Of particular concern, testing rates in CDC-funded testing sites dropped in MSM 18 years or younger from 2012-2017 (10). Despite bacterial STIs being identified as potential drivers of HIV infection (11-15), STI screening rates remain low in this population, with less than half of YMSM reporting STI testing in the last year (16). Barriers to HIV/STI testing include lack of access to testing sites, concerns about privacy, low-perceived risk, and stigma (17-19).
HIV self-testing with rapid results and STI selfcollection (and mailing of samples to a lab for testing) are promising approaches to increase HIV/STI testing rates, reach the undiagnosed, and circumvent common barriers to testing (20). A number of studies have demonstrated the feasibility and acceptability of self-administered HIV/ STI sample collection and testing (21-26). Data suggests that mHealth-based approaches to expanding HIV selftesting and STI self-collection, including web-based portals and mobile apps, are feasible, acceptable and may increase access (27). In a randomized trial evaluating the effect of providing HIV self-tests among 2,665 MSM in the US, those provided quarterly internet-distributed self-HIV test kits were more likely to test 3 or more times during the trial compared with a control group ( $77 \%$ vs. $22 \%)$, with twice the number of new HIV infections identified in the selftesting group (24). MSM have also expressed preferences for self-collection of STI specimens, coupled with electronic delivery of negative STI test results and direct provider notification for positive results (28).

To the best of our knowledge, no study has examined the utility and efficacy of mHealth approaches to HIV self-testing and STI self-collection among YMSM in the US. Given that app use is nearly ubiquitous among young people (29), young people report getting much of their health information through technology $(30,31)$, and sexual health apps have shown acceptability and initial efficacy in reducing HIV risk among YMSM $(32,33)$, integrating HIV self-testing and STI self-collection into a broader sexual health app may be particularly relevant and effective (20). Through the North Carolina/Emory Center for Innovative Technology (iTech) (34), a part of the National Institutes of Health's Adolescent Medicine Trials Network for 
HIV/AIDS Interventions (ATN) (35), our teams have developed and tailored two mobile apps, LYNX (36) and MyChoices (37), to increase HIV/STI testing and preexposure prophylaxis (PrEP) uptake among YMSM. Each of these apps supports the ordering and delivery of a rapid HIV self-test kit and a kit for collection and mailing of samples for bacterial STI testing, with provision of test results to participants. This manuscript will report experiences and lessons learned with HIV self-test and STI self-collection kits ordered via the LYNX or MyChoices app within two pilot randomized controlled trials (RCTs) among YMSM across five US cities.

\section{Methods}

Data from this study comes from two separate protocols within the ATN-LYNX and MyChoices (ATN 140 and 141 , respectively), which have been described previously $(36,37)$. These pilot RCTs were designed to examine the feasibility and acceptability of two separate mobile apps in increasing HIV testing and PrEP initiation among YMSM in the US. While the protocols were distinct, the protocol teams harmonized procedures and measures to facilitate cross-study analyses.

\section{Study population and recruitment}

Eligible participants were cisgender men who (I) were aged 15-24 years; (II) had not tested for HIV in the past 3 months; (III) self-reported being HIV-uninfected or HIV status unknown; (IV) owned an iOS or Android mobile phone and willing/able to download the LYNX or MyChoices app; (V) were fluent in English; (VI) were not taking PrEP; (VII) had self-reported evidence of being at risk for HIV acquisition (36,37); (VIII) had not received experimental HIV vaccine product; (IX) were not currently enrolled in another HIV intervention study; and (X) did not have any condition that would make participation unsafe, complicate interpretation of study outcome data, or interfere with achieving study objectives.

Participants were recruited across five iTech study sites: for LYNX, Chicago, IL (CORE Center) and Tampa, FL (University of South Florida); and for MyChoices, Boston, MA (Fenway Health), the Bronx, NY (Children's Hospital at Montefiore), Chapel Hill, NC (University of North Carolina at Chapel Hill). Recruitment methods included web-based and social media strategies (e.g., Craigslist, social networking ads, and gay sexual networking mobile apps); distributing posters, flyers, and palm cards; direct outreach at local venues frequented by YMSM; clinic-based recruitment; and long-chain referral.

\section{Study procedures}

Study procedures were reviewed and approved by the University of North Carolina Institutional Review Board (IRB) as the single IRB of record, and IRB authorization agreements with all participating research entities were enacted. The study was conducted in accordance with the Declaration of Helsinki (as revised in 2013). A Certificate of Confidentiality was obtained from the National Institute of Child Health and Human Development, and a waiver of parental consent was obtained for participants who were 15-17 years old. The studies were also registered on ClinicalTrials.gov (NCT03177512 and NCT03179319).

All participants underwent screening in a private room at the study site. Eligible participants then participated in an informed consent/assent process.

MyChoices enrolled 60 participants and LYNX enrolled 61 participants ( $\mathrm{N}=121$ across both protocols) who were randomized 2:1 to receive the respective app $(n=40$ per protocol; $n=80$ in total) or standard of care $(n=20-21$ per protocol; $\mathrm{n}=41$ in total) —CDC pamphlets with information on why, how and where to obtain HIV testing and PrEP. Men who were randomized to receive either app were given brief instructions on the purpose of the app, how to access it, and an overview of how to use it. For this analysis, we only present data on the intervention participants who were randomized to receive the mobile apps and had access to HIV self-testing and STI self-collection kits.

\section{Brief description of the mobile apps}

The development and content of both LYNX and MyChoices has been described previously $(36,37)$. Both apps were developed with input from men aged 15-24 via iterative development, testing and adaptation, through interviews, focus groups, theater testing and technical pilots. While the apps were developed by separate research teams (and developers) using different theoretical models to effect behavior change and different approaches for engagement, both LYNX and MyChoices include the ability to order home self-test kits (see Figure 1) for HIV, syphilis, gonorrhea and chlamydia. 
A

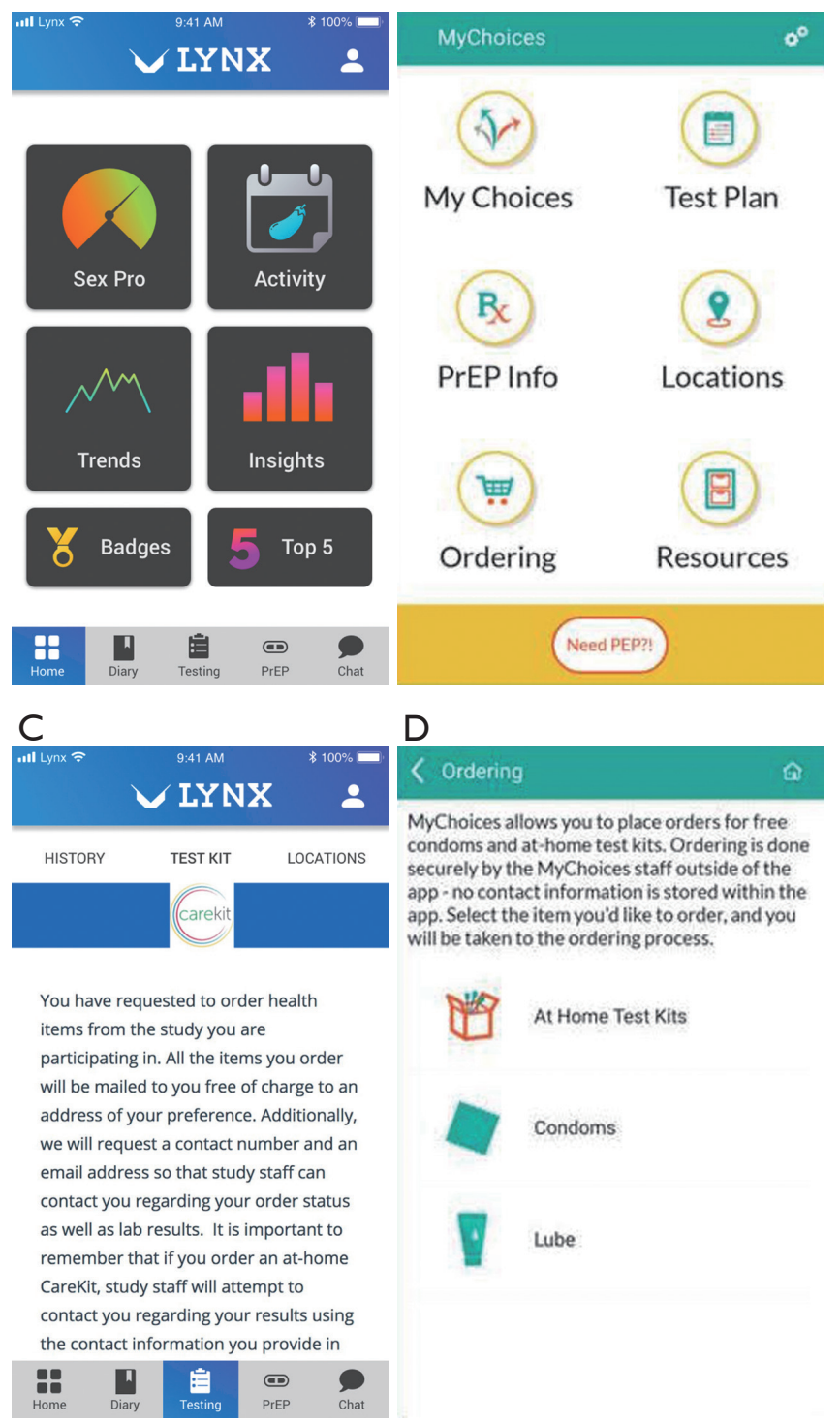

Figure 1 Screenshots from the two mHealth apps: (A) LYNX homepage, (B) MyChoices homepage, (C) LYNX HIV self-test and STI self-collection kit ordering page, (D) MyChoices HIV self-test and STI self-collection kit ordering page. YMSM, young men who have sex with men; STI, sexually transmitted infection.

\section{Description of HIV self-test and STI self-collection kits ordering and processing}

The HIV and STI kits were provided by Emory University's Center for AIDS Research (CFAR) Prevention Science Core. If a participant selected to order either kit through either app, they were taken to a secure online order form to provide their mailing address and contact information.
Orders were fulfilled and shipped through the study's Amazon Multichannel Fulfillment account, and once shipped, arrived within 3-10 business days in a generic Amazon box. Study staff sent an SMS or email to the participant when the package was shipped (with tracking information) and again when it was delivered.

The HIV test kit included one OraQuick swab and tube with solution (Orasure Technologies, Bethlehem, PA, USA), instructions on how to use and read the test and what to do in case of a reactive result (i.e., obtain confirmatory test). It was self-administered using oral fluid as a sample, and results could be read by the participant in 20 minutes.

The STI collection kits contained the following: (I) a welcome letter describing the contents of the kit, who to contact in case of questions, instructions for sample collection including a link to a how-to video, and a link to register their unique ID; (II) sample collection materials, including fingerstick for capillary blood collection for syphilis, and urethral, rectal and pharyngeal swabs for gonorrhea and chlamydia, and tubes pre-labeled with a unique ID; (III) a lab requisition form; and (IV) a prepaid and labeled bubble mailer for shipping specimens back to the lab and mailing instructions. Participants were instructed to complete the lab requisition form, and ship this form along with their collected specimens via FedEx Standard Overnight shipping to a CLIA-certified at Emory University's CFAR. For syphilis, samples were tested using Rapid Plasma Reagin kits (Arlington Scientific Inc., Springville, UT), and for chlamydia and gonorrhea, all swabs were collected using the Abbott Multicollect kits and tested by real-time polymerase chain reaction (Abbott Molecular Inc., Des Plaines, IL). Once specimens were processed (within approximately one week of receipt at the lab), results were provided to the participant via email or the app (if non-reactive) or by the study staff at the enrolling site via phone (if reactive). Treatment for reactive results was facilitated by the enrolling study site.

\section{Measures}

\section{Timing and structure}

Participants completed three self-administered, selfreported study assessments: at baseline/pre-randomization and at 3- and 6-months post-randomization. Participants received US \$50-60 USD for the in-person baseline assessment and \$25-30 USD for each completed webbased follow-up assessment. After the 3-month or 6-month assessment, online interviews were conducted with a 
purposive sample of participants randomized to the app arms $(n=37)$. Participants were selected to ensure diversity in race/ethnicity, age and level of app usage (high and low users).

Sociodemographic variables were assessed at baseline and include: enrollment city, age, race/ethnicity, educational status, and insurance status.

Prior HIV testing history was assessed by asking individuals if they had ever had a prior HIV test. HIV testing self-efficacy at baseline was ascertained by asking eight questions about how confident they are that they could perform certain testing-related behaviors (e.g., getting tested once per year, buying a home test). Participants responded on a 5-point Likert scale ranging from completely confident to not at all confident.

Order tracking and lab measures include number of HIV test kits and STI collection kits ordered through the apps, number of STI kits returned to the lab, and number of positive STI test results (overall and by STI type and infection site).

Self-reported HIV/STI testing measures include reporting any HIV testing, any HIV self-testing, and selfcollection of STI samples (regardless of whether they were returned to the lab) over follow up. Additionally, participants who ordered an STI self-collection kit reported whether they had experienced any STI-related symptoms (e.g., pain, discharge) prior to ordering the kit.

App provision of self-testing and self-collection acceptability measures include difficulty and helpfulness in ordering the kits. Both were measured on a 5-point Likert scale, with responses ranging from "Not at all" to "Extremely".

HIV self-testing acceptability measures were assessed among those who self-reported using an HIV self-test kit during follow-up and include convenience of home testing, confidence that they used the test correctly, and number of people with whom they tested. These were only asked of participants in the LYNX study due to a survey error in the MyChoices study.

STI self-collection acceptability measures were assessed only among those who self-reported ordering an STI selfcollection kit during follow-up. These include convenience of self-collection, satisfaction with level of privacy, comfort with self-collection $v s$. clinical testing and whether they would use self-collection in the future. Those who reported collecting STI samples indicated their level of confidence and any difficulties in using the STI self-collection kits correctly, including following the instructions, opening and using the tubes, collecting samples from each anatomical site and mailing the samples back to the lab. These were measured on a 5 -point Likert scale, with responses ranging from "Not at all" to "Extremely".

For both HIV self-test and STI self-collection kits, participants who did not report ordering a kit were asked to select the reason(s) for not ordering one, including a lack of time to test, not having a comfortable place to self-test/ collect, preference for testing at a clinic, not having a good time to test at home, worried about someone seeing them use the test, recently testing negative somewhere else, and not thinking they were at risk.

Online interview domains included experiences ordering the HIV self-test and STI self-collection kits, feedback on the delivery process, experiences and challenges with using different components of the kits, concerns about privacy, reasons for not ordering or completing the testing process, and suggestions for improving the process.

\section{Data analysis}

Descriptive analyses were conducted to characterize the sample and to illustrate experiences with HIV self-testing and STI self-collection. We report means and standard deviations (SD) for continuous measures and frequencies and proportions for nominal measures.

For prevalence of self-collection and self-testing and for positivity rates, we combined 3 and 6-month follow up data to calculate rates across follow up. For acceptability measures, we used 6-month data for those who completed the 6-month follow up (to capture longest experience with the app and most opportunity for self-collection and selftesting); however, for those who did not have 6-month data, we used 3-month data, if available. Responses did not differ meaningfully or significantly across timepoints.

Lastly, we performed $t$-tests and chi-square tests to determine whether rates of ordering differed by baseline characteristics. However, there were no significant correlates so we do not report on them further here.

The qualitative data from the exit interviews were professionally transcribed. Members of the iTech Analytic Core reviewed all transcripts to identify themes related to HIV self-testing and STI self-collection. Thematic analysis then involved using a primarily deductive approach to synthesize data (38-40). Illustrative quotes were pulled from interviews across both studies. 
Table 1 Baseline characteristics of MyChoices and LYNX pilot RCT app users (N=80), 2018-2019, United States

\begin{tabular}{|c|c|}
\hline Characteristics & $\mathrm{N}[\%]$ \\
\hline \multicolumn{2}{|l|}{ Sociodemographics } \\
\hline Age (range: 16-24), mean (SD) & $20.7(2.4)$ \\
\hline \multicolumn{2}{|l|}{ Study site city } \\
\hline Boston, MA & $20[25]$ \\
\hline Bronx, NY & $6[7]$ \\
\hline Chapel Hill, NC & $14[17]$ \\
\hline Chicago, IL & $22[28]$ \\
\hline Tampa, FL & 18 [23] \\
\hline \multicolumn{2}{|l|}{ Race/ethnicity } \\
\hline Latinx & $17[21]$ \\
\hline Black, non-Latinx & $11[14]$ \\
\hline White, non-Latinx & $41[51]$ \\
\hline Multiracial/other & $11[14]$ \\
\hline Currently in school & $55[70]$ \\
\hline \multicolumn{2}{|l|}{ Highest level of education completed } \\
\hline Less than HS & $13[16]$ \\
\hline High school diploma/GED & $3[4]$ \\
\hline Some college/tech or vocational school & $42[53]$ \\
\hline Four-year college graduate or more & $21[27]$ \\
\hline \multicolumn{2}{|l|}{ Insurance } \\
\hline None & $14[17]$ \\
\hline Public & $14[17]$ \\
\hline Private & $52[65]$ \\
\hline \multicolumn{2}{|l|}{ HIV and STI testing history } \\
\hline Ever prior HIV test & $50[63]$ \\
\hline \multicolumn{2}{|l|}{ HIV testing self-efficacy ${ }^{\dagger}$} \\
\hline $\begin{array}{l}\text { Getting tested for HIV at least once per } \\
\text { year }\end{array}$ & $53[71]$ \\
\hline Getting an HIV test after condomless sex & $40[53]$ \\
\hline Asking my doctor or nurse for an HIV test & $51[68]$ \\
\hline $\begin{array}{l}\text { Discussing condomless sex with a doctor } \\
\text { or nurse }\end{array}$ & $41[55]$ \\
\hline Buying a HIV self-test & $44[59]$ \\
\hline Using a HIV self-test & $48[64]$ \\
\hline Understanding the results of a HIV self-test & 54 [72] \\
\hline
\end{tabular}

Table 1 (continued)
Table 1 (continued)

\begin{tabular}{lc}
\hline Characteristics & N [\%] \\
\hline $\begin{array}{l}\text { Knowing how long to wait after condomless } \\
\text { sex before getting tested for HIV }\end{array}$ & $40[53]$ \\
Ever prior STI test & $44[59]$ \\
Tested in the past 3 months & $2[3]$ \\
STI testing frequency (among prior testers) & \\
Every 2-4 months & $3[7]$ \\
Every 6 months & $8[18]$ \\
Every 8-12 months & $9[20]$ \\
No regular testing frequency & $24[55]$ \\
\hline
\end{tabular}

Note: rows may not sum to $100 \%$ due to rounding error. ${ }^{\dagger}$, completely/very confident vs. somewhat/not very/not at all confident. STI, sexually transmitted infection; RCT, randomized controlled trial.

\section{Results}

Among the 80 participants enrolled and randomized to the intervention arm across both studies, 67 (84\%) completed the 3 -month follow up assessment and $63(79 \%)$ completed the 6-month follow up assessment, resulting in 71 (89\%) who completed at least one follow up assessment.

Baseline characteristics are described in Table 1. In brief, participants were on average 20.7 years of age $(S D=2.4$; range: $16-24)$, and $49 \%$ were non-White or multiracial (including 21\% Latinx and 14\% Black). Nearly two-thirds (63\%) had had a prior HIV test. Over half $(58 \%)$ had a prior STI test, but only $3 \%$ had tested for STIs within the past 3 months; $55 \%$ reported that they did not have a regular frequency for STI testing.

\section{HIV self-testing and STI self-collection rates: lab and self- report measures}

A majority (76\%) of those who had a follow up survey reported having an HIV test during study follow up, and 54\% reported using at least one HIV self-test kit during this time period. HIV self-test kits accounted for $70 \%$ of all HIV tests during this period (Table 2). Specifically, among those who reported having an HIV test over follow up, $24 \%$ only tested at a clinical or community-based site, $24 \%$ tested at a clinical or community-based site AND with a self-test kit, and $52 \%$ only tested with a self-test kit. According to the order tracking reports, $65 \%$ ordered at least one HIV self-test kit over the course of the study 
Table 2 HIV self-testing and STI self-collection over follow up of MyChoices and LYNX pilot RCT app users (N=80), 2018-2019, United States

\begin{tabular}{lcc}
\hline HIV self-testing and STI self-collection & N & $\%$ \\
\hline Had at least one HIV test (any kind), self-report & 54 & $76^{\dagger}$ \\
Ordered at least one HIV Test, order tracking & 52 & $65^{\ddagger}$ \\
Used at least one HIV self-test, self-report & 38 & $54^{\dagger}$ \\
Ordered at least one STI kit, order tracking & 43 & $54^{\ddagger}$ \\
Collected at least one STI sample, self-report & 25 & \\
Urine & 15 & 60 \\
Throat & 24 & 96 \\
Rectal & 14 & 56 \\
Blood & 15 & 60 \\
Returned at least one STI kit, lab report & 10 & $13^{\ddagger}$ \\
Positive/returned STI results, lab report & $5 / 94$ & 5 \\
Syphilis & $2 / 14$ & 14 \\
CT, urethral & $1 / 13$ & 8 \\
CT, pharyngeal & $1 / 13$ & 8 \\
CT, rectal & $0 / 13$ & 0 \\
GC, urethral & $0 / 13$ & 0 \\
GC, pharyngeal & $0 / 14$ & 0 \\
GC, rectal & $1 / 14$ & 7 \\
\hline, percent of thos & & \\
\hline
\end{tabular}

${ }^{\dagger}$, percent of those with at least one follow-up assessment $(\mathrm{N}=71) ;{ }^{\ddagger}$, percent of all participants $(\mathrm{N}=80)$. STI, sexually transmitted infection; RCT, randomized controlled trial; CT, chlamydia; GC, gonorrhea.

(range: $0-4$, mean $=0.86, \mathrm{SD}=0.87$ ). On average, HIV selftest kits were delivered to participants 6 days after placing their order. Although we do not have precise usage data for all participants who ordered the HIV self-test kit through the app, among the participants who had any follow up data, $68 \%$ ordered an HIV test according to the order tracking data and $75 \%$ of these self-reported using an HIV self-test during the study.

According to the order tracking and lab reports, 54\% ordered at least one STI self-collection kit (range: 0-4, mean $=0.71, \mathrm{SD}=0.84$ ) over the course of follow up (Table 2), and on average, STI self-collection kits were delivered to participants less than 6 days after placing their order. Notably, $68 \%$ of participants self-reported ordering at least one STI self-collection kit, of which $52 \%(n=25)$ reported collecting at least one sample (24 pharyngeal, 15 urine,
15 blood and 14 rectal). Only four participants reported experiencing any STI symptoms prior to ordering the kit. According to lab data, 10 participants returned at least one STI sample (14 kits and 94 samples in total), of which there were 5 unique reactive tests (for 4 unique individuals), resulting in a $5 \%$ positivity rate (5/94 samples) among $40 \%$ (4/10) of participants who returned the samples. See Table 2 for prevalence by STI (chlamydia, gonorrhea, syphilis) and anatomical site. On average, results were delivered to the participant approximately 10 days after sample collection.

\section{App acceptability measures}

Among all app users who reported using the ordering feature, $93 \%$ reported that it was "not at all" $(83 \%)$ or only "a little" (9\%) difficult to order HIV self-test and/or STI self-collection kits through the app. Notably, only 1 participant reported it being "very" difficult. Similarly, 87\% of app users reported that it was "extremely" (71\%) or "very" (15\%) helpful to be able to order these kits and other safer sex supplies (i.e., condoms and lube) through the app.

\section{HIV self-testing acceptability measures (among LYNX participants only)}

Among the 20 participants who reported using an HIV selftest in the LYNX study, 63\% reported testing alone, $26 \%$ with one other person and $11 \%$ with two other people. Moreover, $80 \%$ reported that it would be very convenient to use in the future and that they were extremely confident in using the test correctly (Table 3).

Among those who reported not ordering a HIV selftest kit $(n=13)$, the most commonly endorsed reasons were that they would rather test at a clinic $(\mathrm{n}=7 ; 54 \%)$, had a recent negative test somewhere else $(n=4 ; 31 \%)$ and did not think they were at risk $(n=4 ; 31 \%)$, followed by not having the time to test in general $(n=3 ; 23 \%)$. Less commonly endorsed reasons included difficulty finding a time to test at home ( $\mathrm{n}=1 ; 8 \%)$, worrying about someone seeing them use the test $(n=1 ; 8 \%)$ and having a recent positive test $(n=1 ; 8 \%)$ (notably, no one reported a positive test result in follow up surveys).

\section{STI self-collection acceptability measures}

STI self-collection was highly acceptable for those who reported ordering a kit through the app. Over threequarters responded that they "agree" or "strongly agree" 
STI self-collection is convenient (78\%), that their privacy was protected throughout the process $(83 \%)$ and that they would use STI self-collection in the future $(85 \%)$. Just over half reported that they felt more comfortable collecting their own samples compared to a doctor (51\%) (Table 4).

Among those who reported ordering and collecting STI samples using the self-collection kits, $80 \%$ were extremely $(36 \%)$ or very $(44 \%)$ confident that they did the STI collection correctly. Most found that the separate components of the kit were "not at all" or "only a little difficult" (Table 5); however, 50\% found collecting the blood sample difficult.

Table 3 HIV self-test kit acceptability among LYNX participants who reported using a HIV self-test over follow up $(n=19)^{\dagger}$

\begin{tabular}{lc}
\hline HIV self-test kit acceptability & $\mathrm{N}[\%]$ \\
\hline Convenience of using test kit in future & $16[80]$ \\
Very convenient & 0 \\
Convenient & $1[5]$ \\
Neutral & 0 \\
Inconvenient & $3[15]$ \\
Very inconvenient & \\
Confidence in correct use of test & $16[80]$ \\
Extremely confident & $3[15]$ \\
Confident & $1[5]$ \\
Neutral & 0 \\
Not very confident & 0 \\
Not confident at all & \\
\hline
\end{tabular}

${ }^{\dagger}$, data were not collected for MyChoices participants.
Among those who reported not ordering a STI selfcollection kit $(n=39)$ and had follow up data $(n=33)$, the most commonly endorsed reasons were that they would rather test at a clinic $(\mathrm{n}=13 ; 45 \%)$ and did not think they were at risk ( $\mathrm{n}=12 ; 41 \%)$, followed by not having the time to test in general $(\mathrm{n}=8 ; 28 \%)$ and recently testing negative somewhere else $(n=5 ; 17 \%)$. Less commonly endorsed reasons for not ordering a STI self-collection kit included finding a hard time to test at home $(\mathrm{n}=3 ; 10 \%)$, worry about someone seeing them use the test $(\mathrm{n}=2 ; 7 \%)$ and not having a comfortable place to test $(\mathrm{n}=1 ; 3 \%)$.

\section{Qualitative feedback from online interviews}

Almost all participants found the ordering process on both apps easy and clear. They liked having tracking information to closely predict delivery. Kits were delivered in unmarked packaging and participants appreciated the privacy that provided, though most said they were not worried about anyone in their home seeing the package.

The majority of participants found the HIV self-test kit easy to use. Many followed the included instructions or app-based instructional videos, and felt they were helpful and made the process easy. They felt confident in the results of their HIV self-test and some preferred it over going to a clinical site.

"...the HIV one was literally just very quick direction, super friendly, super effective."

When it came to the STI self-collection kit, the experiences were more mixed. Participants who ordered and used the STI self-collection kit felt that testing themselves for STIs gave them control over their sexual health.

"I just felt it was like--again, the feeling of like baving agency

Table 4 STI Self-collection kit acceptability among MyChoices and LYNX participants who self-reported ordering an STI self-collection kit over follow up $(\mathrm{n}=41)$

\begin{tabular}{|c|c|c|c|c|c|}
\hline STI self-collection kit acceptability & $\begin{array}{c}\text { Strongly } \\
\text { agree, N [\%] }\end{array}$ & Agree, $\mathrm{N}[\%]$ & Neither, $\mathrm{N}$ [\%] & $\begin{array}{c}\text { Disagree, } \mathrm{N} \\
{[\%]}\end{array}$ & $\begin{array}{c}\text { Strongly } \\
\text { disagree, N [\%] }\end{array}$ \\
\hline $\begin{array}{l}\text { Home testing is convenient way to get STI } \\
\text { testing }\end{array}$ & $17[41]$ & $15[37]$ & $4[10]$ & $1[2]$ & $5[10]$ \\
\hline $\begin{array}{l}\text { Felt like my privacy was protected throughout } \\
\text { testing process }\end{array}$ & 19 [46] & $15[37]$ & 5 [12] & 0 & $2[5]$ \\
\hline I would use home-based testing in the future & $21[51]$ & 14 [34] & $5[12]$ & $1[2]$ & 0 \\
\hline
\end{tabular}

Note: columns may not sum to $100 \%$ due to rounding error. STI, sexually transmitted infection. 
Table 5 Difficulty of STI self-collection kit use among MyChoices and LYNX participants who self-reported ordering and using an STI selfcollection kit over follow up $(\mathrm{n}=25)$

\begin{tabular}{|c|c|c|c|c|c|}
\hline STI self-collection usage experience & $\begin{array}{l}\text { Not at all, } \\
\quad \mathrm{N}[\%]\end{array}$ & A little, $N$ [\%] & $\begin{array}{c}\text { Somewhat, } \\
\text { N [\%] }\end{array}$ & Very, N [\%] & $\begin{array}{c}\text { Extremely, } \\
\mathrm{N}[\%]\end{array}$ \\
\hline How confident that did the STI self-collection correctly & $1[4]$ & $1[4]$ & $3[12]$ & $11[44]$ & $9[36]$ \\
\hline Do STI self-collection, overall & $10[40]$ & $14[56]$ & 0 & $1[4]$ & 0 \\
\hline Collect rectal swab & 7 [39] & 7 [39] & $3[17]$ & $1[6]$ & 0 \\
\hline Collect throat swab & 9 [36] & $11[44]$ & $4[16]$ & $1[4]$ & 0 \\
\hline Collect urine sample & $10[56]$ & 6 [33] & $2[11]$ & 0 & 0 \\
\hline
\end{tabular}

Note: columns may not sum to $100 \%$ due to rounding error. STI, sexually transmitted infection.

over. Like what was being tested like what I was being tested over and the fact that I was the one conducting it."

Additionally, many appreciated the included instructions and in-app videos and liked how easy it was to collect samples at home and drop the pre-paid package into the mail.

"It was good, I really like the step by step instruction and the fact that it was like in each bag. Its own like--testing whatever it was testing like it was--it had its own instructions, instead of like being in a book with it."

Among the participants who used the STI kits, most described the swab collections as straightforward; though, some explained that it did take them a while to complete all the samples, and that they asked for support from a friend or study site staff. Several participants expressed frustration with the blood sample process for syphilis. They had trouble getting blood into the tube or getting as much blood as was required. Some participants sent back incomplete blood samples or skipped the blood collection all together. One participant was so frustrated by the blood sample that he didn't finish the rest of the self-collection kit.

"So I just kind of, I was definitely discouraged in that moment. So I just kind of like put it away and like put the box away and I was like, I'll get another kit eventually"

A few participants who returned kits expressed distress around getting results and would have liked a clearer timeline of when to expect results and how they should expect to receive them. Some participants described being accustomed to quicker shipping and turnaround times and therefore became worried when it took longer than anticipated to get their kit or results.

Of the participants who ordered but did not return kits, many cited confusion around kit instructions or being overwhelmed with the process. Some of the participants who found the kit overwhelming said they were afraid of doing something wrong and getting incorrect results.

"Like, what, like, what am I supposed to-I didn't know why I got confused honestly, but, like, I just, like, kind of, like, kept putting them off, because I was, like, how, like, I don't want to do this wrong."

Additionally, others cited a lack of time to complete all the steps, which, in a few instances, contributed to a period of many days between sample collection and return, causing concern in some participants that results might be invalid.

\section{Discussion}

This appears to be the first study to evaluate the HIV selftesting and STI self-collection via mobile apps among YMSM. This mixed-methods study indicated that both HIV self-testing and STI self-collection was highly acceptable. Of those who had access to the ordering page on either app, approximately two-thirds ordered a HIV self-test, and three-fourths of these individuals used the tests. Moreover, 
of the individuals who had an HIV test at all over follow up, half used only a self-test and an additional quarter used both a self-test and a clinic or community-based test. High enthusiasm and rates of acceptability have been shown in prior studies and among diverse populations (20). However, rates of HIV self-testing were substantially higher in this study compared to what has been reported among adult MSM in the US. In an online national survey of over 2,500 MSM, approximately $10 \%$ had used a HIV self-test kit in the prior 12 months (41). Notably, in this same study, an intervention to compare the provision of self-test kits compared to standard of care, $96 \%$ of intervention arm had at least one test compared to $63 \%$ in the control arm (24). In another trial within emergency departments (ED), $48 \%$ of individuals provided an HIV self-test kit after refusing a HIV test in the ED used the kit -8 times higher than those who only received clinic-based referrals $(\mathrm{RR}=8.45$; 95\% CI: 2.09-34.17) (42). The growing evidence suggests that HIV self-testing is not only highly acceptable but also increases HIV testing, an essential first step in both the HIV treatment and prevention cascades.

STI self-collection was also acceptable, which has been shown in other studies $(28,43)$, but experiences were more mixed. Fewer ordered and used the STI self-collection kits compared to the HIV self-test kits. Specifically, approximately half of the sample ordered a STI self-collection kit; among those only approximately half reported collecting at least one sample and less than a quarter returned the STI samples to the lab for processing. This resulted in $13 \%$ of participants getting results from the STI test kits. This is much lower than reported in a survey in the United Kingdom, which found that $45 \%$ of all individuals who had an STI test used a self-test kit, and $73 \%$ of them returned sufficient samples for processingalthough just over half of those aged 16-20 years returned the kits. In the current study, while over half of the cohort had been tested for STIs in the past, only $3 \%$ had tested in the past 3 months at baseline. Participants cited multiple barriers to using the STI kits during the study, including lack of time, confusion around kit instructions, and concerns about accuracy of test results with delays in returning kits after specimen collection. Importantly, among those who did return the sample, the positivity rate was high $-40 \%$ of individuals had at least one reactive sample, suggesting potential self-selection for higher risk individuals. Furthermore, the high STI prevalence but limited uptake of STI self-collection suggests that further interventions to better educate youth in self-collection and in the high burden of asymptomatic STI among YMSM are warranted.

The process of ordering the kits through the app was also highly acceptable, with approximately $90 \%$ reporting that they did not have difficulty ordering the kits through the app and found this function very helpful. The most common reasons reported for not ordering the kits included a preference for clinic-based testing, low self-perceived HIV risk, and not having time to test. These findings suggest that clinic and self-testing are complementary (44). However, given that over half of our cohort only tested at home during this study, our findings suggest that HIV self-testing and STI self-collection has great potential to increase the proportion and frequency of testing among YMSM. Prior studies have shown that innovative uses of technology can effectively promote self-testing (45-50). Moreover, by combining the facilitation of access to HIV self-test and STI self-collection kits with provision of information about risk behaviors and functionality to improve risk perception among its young users, the potential for expanding access to HIV/STI testing may be further increased.

Based on our findings, we recommend several improvements to the HIV self-testing and STI selfcollection process to address challenges encountered with using kits via our mobile apps. First, youth may benefit from check-ins from staff on whether they are experiencing any problems with ordering kits and using their ordered kits, as well as reminders to send the kits back for processing. This communication could occur via a chat or communication feature within the apps. As the blood sampling process was the most challenging, additional support for the collection of blood (e.g., access to real-time video conferencing support) may be helpful to increase successful completion rates. Second, because several participants expressed concerns about getting their results, providing additional tracking information about specimens returned to the lab and the status and timing of results would help alleviate their worries. Finally, as participants were provided information in writing that they should mail samples within 24 hours of collection, several participants were concerned about inaccurate results due to delays in specimen return after collection; providing additional guidance around required timelines, including the maximum number of days, for mailing in samples might increase kit return rates.

This study has the following limitations. First, this is a convenience sample of YMSM with substantial sexual risk and suboptimal HIV testing, and as such may not be generalizable to the broader population of YMSM. 
Importantly, this study did include a racially and ethnically diverse group of young people from five US cities (including three distinct regions-Northeast, Midwest and Southeast). Additionally, this study only followed individuals for up to six months, so data on repeat testing is limited. Future studies should include a longer follow-up period. Also, because the HIV test kits did not have to be returned to the lab for results, we must rely on self-report for actual kit usage (51). Future studies might explore other methods of validating HIV test usage and results, including uploading a photo to an app. There were also some discrepancies between ordering/lab reports and self-reported ordering of STI kits, which may reflect social desirability, misreading the question, or incomplete online orders. Lastly, because of the sample size, we were limited in our ability to do stratified analyses. Given these limitations, this study is the first to describe HIV self-testing and STI self-collection within two distinct mobile apps among YMSM in the U.S.

In conclusion, we demonstrated that ordering, collection and testing for HIV and other bacterial STIs via mobile apps is feasible and acceptable among YMSM, a highly vulnerable population with the lowest testing rates nationally. Based on the favorable results of this study, the LYNX and MyChoices apps are currently being tested in an efficacy trial across a broader range of US sites (NCT03965221). If successful, these innovative mobile health approaches could be rapidly scaled up to increase HIV/STI testing among youth across the US.

\section{Acknowledgments}

We would like to acknowledge the following people for their support implementing the MyChoices and LYNX studies: Jessica Roberts, Maria Zlotorzynska, Ryan Muench, Pedro Alonso Serrano, Amayvis Garcia, Elizabeth Enriquez-Bruce, Jonathan Hill-Rorie, Zach Soberano.

Funding: NIH Adolescent Trials Network (U01HD068040; PI: Hightown-Weidman/Sullivan); Emory Center for AIDS Research (P30AI050409; PI: del Rio) for Carekit support; MAC AIDS Fund.

\section{Footnote}

Provenance and Peer Review: This article was commissioned by the Guest Editor (Lisa Hightow-Weidman) for the series "Technology-based Interventions in HIV Prevention and Care Continuum among American Youth" published in $m$ Health. The article has undergone external peer review.
Data Sharing Statement: Available at http://dx.doi. org/10.21037/mhealth-20-70

Conflicts of Interest: All authors have completed the ICMJE uniform disclosure form (available at http://dx.doi. org/10.21037/mhealth-20-70). The series "Technologybased Interventions in HIV Prevention and Care Continuum among American Youth" was commissioned by the editorial office without any funding or sponsorship. LHW served as the unpaid Guest Editor of the series. $\mathrm{KBB}, \mathrm{CH}, \mathrm{KHM}, \mathrm{SM}$, JD, JN, LHW, KB and DF reports grants from NICHD, during the conduct of the study. PS reports grants from NIH, during the conduct of the study; and grants and personal fees from NIH, CDC, and Gilead Sciences, personal fees from Merck, and Ontario HIV Research Network, outside the submitted work. SB reports grants from NICHD, during the conduct of the study; non-financial support from Gilead Sciences, outside the submitted work. AL reports grants from NICHD, during the conduct of the study; grants and other from Gilead Sciences, grants from Viiv Healthcare, outside the submitted work. The authors have no other conflicts of interest to declare.

Ethical Statement: The authors are accountable for all aspects of the work in ensuring that questions related to the accuracy or integrity of any part of the work are appropriately investigated and resolved. Study procedures were reviewed and approved by the University of North Carolina Institutional Review Board (IRB) as the single IRB of record, and IRB authorization agreements with all participating research entities were enacted. The study was conducted in accordance with the Declaration of Helsinki (as revised in 2013). A Certificate of Confidentiality was obtained from the National Institute of Child Health and Human Development, and a waiver of parental consent was obtained for participants who were 15-17 years old. All participants participated in an informed consent/assent process.

Open Access Statement: This is an Open Access article distributed in accordance with the Creative Commons Attribution-NonCommercial-NoDerivs 4.0 International License (CC BY-NC-ND 4.0), which permits the noncommercial replication and distribution of the article with the strict proviso that no changes or edits are made and the original work is properly cited (including links to both the formal publication through the relevant DOI and the license). 
See: https://creativecommons.org/licenses/by-nc-nd/4.0/.

\section{References}

1. Centers for Disease Control and Prevention. HIV and Youth. Available online: https://www.cdc.gov/hiv/group/ age/youth/index.html. Accessed January 25, 2020.

2. Zanoni BC, Mayer KH. The adolescent and young adult HIV cascade of care in the United States: exaggerated health disparities. AIDS Patient Care STDS 2014;28:128-35.

3. Beer L, Mattson CL, Bradley H, et al. Trends in ART Prescription and Viral Suppression Among HIV-Positive Young Adults in Care in the United States, 2009-2013. J Acquir Immune Defic Syndr 2017;76:e1-6.

4. Lally MA, van den Berg JJ, Westfall AO, et al. HIV Continuum of Care for Youth in the United States. J Acquir Immune Defic Syndr 2018;77:110-7.

5. Centers for Disease Control and Prevention. STDs in Men Who Have Sex With Men. Available online: https://www. cdc.gov/std/stats17/msm.htm. Accessed January 25, 2020.

6. Centers for Disease Control and Prevention. STDs in Adolescents and Young Adults. Available online: https:// www.cdc.gov/std/stats17/adolescents.htm. Accessed January 25, 2020.

7. DiNenno EA, Prejean J, Irwin K, et al. Recommendations for HIV Screening of Gay, Bisexual, and Other Men Who Have Sex with Men - United States, 2017. MMWR Morb Mortal Wkly Rep 2017;66:830-2.

8. Van Handel M, Kann L, Olsen EO, et al. HIV Testing Among US High School Students and Young Adults. Pediatrics 2016;137:e20152700.

9. Zlotorzynska M, Sullivan P, Sanchez T. The Annual American Men's Internet Survey of Behaviors of Men Who Have Sex With Men in the United States: 2016 Key Indicators Report. JMIR Public Health Surveill 2019;5:e11313.

10. Wang G, Belcher L, Song W, et al. editors. HIV testing, diagnosis, and linkage to care and prevention services among persons tested in CDC-funded sites, 2012-2017. 2019 National HIV Prevention Conference; 2019 March 18-21, 2019; Atlanta.

11. Bernstein KT, Marcus JL, Nieri G, et al. Rectal gonorrhea and chlamydia reinfection is associated with increased risk of HIV seroconversion. J Acquir Immune Defic Syndr 2010;53:537-43.

12. Koblin BA, Husnik MJ, Colfax G, et al. Risk factors for HIV infection among men who have sex with men. AIDS
2006;20:731-9.

13. Buchbinder SP, Vittinghoff E, Heagerty PJ, et al. Sexual Risk, Nitrite Inhalant Use, and Lack of Circumcision Associated With HIV Seroconversion in Men Who Have Sex With Men in the United States. J Acquir Immune Defic Syndr 2005;39:82-9.

14. Katz DA, Dombrowski JC, Bell TR, et al. HIV Incidence Among Men Who Have Sex With Men After Diagnosis With Sexually Transmitted Infections. Sex Transm Dis 2016;43:249-54.

15. Jones J, Weiss K, Mermin J, et al. Proportion of Incident Human Immunodeficiency Virus Cases Among Men Who Have Sex With Men Attributable to Gonorrhea and Chlamydia: A Modeling Analysis. Sex Transm Dis 2019;46:357-63.

16. Centers for Disease Control and Prevention. HIV Risk, Prevention, and Testing Behaviors -- National HIV Behavioral Surveillance System: Men Who Have Sex with Men, 20 U.S. Cities, 2011. In: HIV Surveillance Special Report 8. Available online: http://www.cdc.gov/hiv/library/ reports/surveillance/\#special. Accessed January 26, 2020.

17. Estem KS, Catania J, Klausner JD. HIV Self-Testing: a Review of Current Implementation and Fidelity. Curr HIV/AIDS Rep 2016;13:107-15.

18. MacKellar DA, Valleroy LA, Secura GM, et al. Perceptions of lifetime risk and actual risk for acquiring HIV among young men who have sex with men. AIDS Behav 2007;11:263-70.

19. Dorell CG, Sutton MY, Oster AM, et al. Missed opportunities for HIV testing in health care settings among young African American men who have sex with men: implications for the HIV epidemic. AIDS Patient Care STDS 2011;25:657-64.

20. Steehler K, Siegler AJ. Bringing HIV Self-Testing to Scale in the United States: a Review of Challenges, Potential Solutions, and Future Opportunities. J Clin Microbiol 2019;57:e00257-19.

21. Lunny C, Taylor D, Hoang L, et al. Self-Collected versus Clinician-Collected Sampling for Chlamydia and Gonorrhea Screening: A Systemic Review and MetaAnalysis. PLoS One 2015;10:e0132776.

22. Conway DP, Guy R, Davies SC, et al. Rapid HIV Testing Is Highly Acceptable and Preferred among High-Risk Gay And Bisexual Men after Implementation in Sydney Sexual Health Clinics. PLoS One 2015;10:e0123814.

23. Figueroa C, Johnson C, Verster A, et al. Attitudes and Acceptability on HIV Self-testing Among Key Populations: A Literature Review. AIDS Behav 2015;19:1949-65. 
24. MacGowan RJ, Chavez PR, Borkowf CB, et al. Effect of Internet-Distributed HIV Self-tests on HIV Diagnosis and Behavioral Outcomes in Men Who Have Sex With Men: A Randomized Clinical Trial. JAMA Intern Med 2020;180:117-25.

25. Mustanski B, Feinstein BA, Madkins K, et al. Prevalence and Risk Factors for Rectal and Urethral Sexually Transmitted Infections From Self-Collected Samples Among Young Men Who Have Sex With Men Participating in the Keep It Up! 2.0 Randomized Controlled Trial. Sex Transm Dis 2017;44:483-8.

26. Sullivan PS, Driggers R, Stekler JD, et al. Usability and Acceptability of a Mobile Comprehensive HIV Prevention App for Men Who Have Sex With Men: A Pilot Study. JMIR Mhealth Uhealth 2017;5:e26.

27. Ko JS, Stafylis C, Klausner JD. Mobile health promotion of human immunodeficiency virus self-testing in the United States. mHealth 2020;6:10.

28. Cushman TA, Graves SK, Little SJ. Attitudes and Preferences Regarding the Use of Rapid Self-Testing for Sexually Transmitted Infections and HIV in San Diego Area Men Who Have Sex With Men. Open Forum Infect Dis 2019;6:ofz043.

29. Clement J. Share of mobile device owners in the United States who download apps at least once a month or more as of April 2018, by age group. Statista. 2019. Available online: https://www.statista.com/statistics/243794/usadult-cell-phone-owners-who-have-downloaded-apps-byage-group/

30. Hightow-Weidman LB, Muessig KE, Bauermeister J, et al. Youth, Technology, and HIV: Recent Advances and Future Directions. Curr HIV/AIDS Rep 2015;12:500-15.

31. Pingel ES, Thomas L, Harmell C, et al. Creating comprehensive, youth centered, culturally appropriate sex education: What do young gay, bisexual and questioning men want? Sex Res Social Policy 2013;10:10.1007/s13178013-0134-5.

32. Bauermeister JA, Pingel ES, Jadwin-Cakmak L, et al. Acceptability and preliminary efficacy of a tailored online HIV/STI testing intervention for young men who have sex with men: the Get Connected! program. AIDS Behavior 2015;19:1860-74.

33. Lelutiu-Weinberger C, Pachankis JE, Gamarel KE, et al. Feasibility, Acceptability, and Preliminary Efficacy of a Live-Chat Social Media Intervention to Reduce HIV Risk Among Young Men Who Have Sex With Men. AIDS Behavior 2015;19:1214-27.

34. Hightow-Weidman LB, Muessig K, Rosenberg E, et al. University of North Carolina/Emory Center for Innovative Technology (iTech) for Addressing the HIV Epidemic Among Adolescents and Young Adults in the United States: Protocol and Rationale for Center Development. JMIR Res Protoc 2018;7:e10365.

35. Lee S, Kapogiannis BG, Allison S. Improving the Youth HIV Prevention and Care Continuums: The Adolescent Medicine Trials Network for HIV/AIDS Interventions. JMIR Res Protoc 2019;8:e12050.

36. Liu A, Coleman K, Bojan K, et al. Developing a Mobile App (LYNX) to Support Linkage to HIV/Sexually Transmitted Infection Testing and Pre-Exposure Prophylaxis for Young Men Who Have Sex With Men: Protocol for a Randomized Controlled Trial. JMIR Res Protoc 2019;8:e10659.

37. Biello KB, Marrow E, Mimiaga MJ, et al. A Mobile-Based App (MyChoices) to Increase Uptake of HIV Testing and Pre-Exposure Prophylaxis by Young Men Who Have Sex With Men: Protocol for a Pilot Randomized Controlled Trial. JMIR Res Protoc 2019;8:e10694.

38. Ryan GW, Bernard HR. Techniques to identify themes. Field methods 2003;15:85-109.

39. Basics of Qualitative Research (3rd ed.): Techniques and Procedures for Developing Grounded Theory. Thousand Oaks, California: SAGE Publications, Inc.; 2008.

40. Johnson BD, Dunlap E, Benoit E. Organizing "mountains of words" for data analysis, both qualitative and quantitative. Subst Use Misuse 2010;45:648-70.

41. Chavez P, MacGowan R, Borkowf C, et al. editors. Characteristics associated with HIV self-testing reported by internet-recruited MSM in the United States, eSTAMP baseline data, 2015. 9th IAS Conference on HIV Science; 2017; Paris, France.

42. Patel AV, Abrams SM, Gaydos CA, et al. Increasing HIV testing engagement through provision of home HIV self-testing kits for patients who decline testing in the emergency department: a pilot randomisation study. Sex Transm Infect 2019;95:358-60.

43. Siegler AJ, Mayer KH, Liu AY, et al. Developing and Assessing the Feasibility of a Home-based Preexposure Prophylaxis Monitoring and Support Program. Clin Infect Dis 2019;68:501-4.

44. Merchant RC, Clark MA, Liu T, et al. Comparison of Home-Based Oral Fluid Rapid HIV Self-Testing Versus Mail-in Blood Sample Collection or Medical/Community HIV Testing By Young Adult Black, Hispanic, and White MSM: Results from a Randomized Trial. AIDS Behavior 2018;22:337-46. 
45. Cleary M, O'Sullivan J. P145 London sexual health transformation programme. Sex Transm Infect 2017;93.

46. Ahmed-Little Y, Bothra V, Cordwell D, et al. Attitudes towards HIV testing via home-sampling kits ordered online (RUClear pilots 2011-12). J Public Health (Oxf) 2016;38:585-90.

47. Kelvin EA, George G, Mwai E, et al. A Randomized Controlled Trial to Increase HIV Testing Demand Among Female Sex Workers in Kenya Through Announcing the Availability of HIV Self-testing Via Text Message. AIDS Behav 2019;23:116-25.

48. De Boni RB, Veloso VG, Fernandes NM, et al. An Internet-Based HIV Self-Testing Program to Increase HIV Testing Uptake Among Men Who Have Sex With

doi: $10.21037 /$ mhealth-20-70

Cite this article as: Biello KB, Horvitz C, Mullin S, Mayer KH, Scott H, Coleman K, Dormitzer J, Norelli J, HightowWeidman L, Sullivan P, Mimiaga MJ, Buchbinder S, Bojan K, Futterman D, Emmanuel P, Liu A; on behalf of the iTech Team. HIV self-testing and STI self-collection via mobile apps: experiences from two pilot randomized controlled trials of young men who have sex with men. mHealth 2021;7:26.
Men in Brazil: Descriptive Cross-Sectional Analysis. J Med Internet Res 2019;21:e14145.

49. Dukers-Muijrers NH, Theunissen KA, Wolffs PT, et al. Acceptance of Home-Based Chlamydia Genital and Anorectal Testing Using Short Message Service (SMS) in Previously Tested Young People and Their Social and Sexual Networks. PLoS One 2015;10:e0133575.

50. Kersaudy-Rahib D, Lydie N, Leroy C, et al. Chlamyweb Study II: a randomised controlled trial (RCT) of an online offer of home-based Chlamydia trachomatis sampling in France. Sex Transm Infect 2017;93:188-95.

51. Tahlil KM, Ong JJ, Rosenberg NE, et al. Verification of HIV Self-Testing Use and Results: A Global Systematic Review. AIDS Patient Care STDS 2020;34:147-56. 